\title{
Rethinking antiparticles. Hermann Weyl's contribution to neutrino physics
}

\author{
Silvia De Bianchi \\ Department of Philosophy, Building B - Campus UAB, Universitat Autònoma de Barcelona 08193, Bellaterra Barcelona, Spain
}

\section{A R T I C L E I N F O}

\section{Article history:}

Received 18 February 2016

Received in revised form

29 March 2017

Accepted 31 March 2017

Available online 9 May 2017

\section{Keywords:}

Weyl

Majorana

two-component theory

neutrino physics

parity

weak interaction

\begin{abstract}
A B S T R A C T
This paper focuses on Hermann Weyl's two-component theory and frames it within the early development of different theories of spinors and the history of the discovery of parity violation in weak interactions. In order to show the implications of Weyl's theory, the paper discusses the case study of Ettore Majorana's symmetric theory of electron and positron (1937), as well as its role in inspiring Case's formulation of parity violation for massive neutrinos in 1957. In doing so, this paper clarifies the relevance of Weyl's and Majorana's theories for the foundations of neutrino physics and emphasizes which conceptual aspects of Weyl's approach led to Lee's and Yang's works on neutrino physics and to the solution of the theta-tau puzzle in 1957. This contribution thus sheds a light on the alleged "re-discovery" of Weyl's and Majorana's theories in 1957, by showing that this did not happen all of a sudden. On the contrary, the scientific community was well versed in applying these theories in the 1950s on the ground of previous studies that involved important actors in both Europe and United States.
\end{abstract}

(๑) 2017 Elsevier Ltd. All rights reserved.

\section{Introduction}

"Everything comes to him who knows how to wait". ${ }^{1}$ By echoing Leo Tolstoy, Wolfgang Pauli gratefully acknowledged the discovery of the neutrino in 1956, the hypothetical particle he had to postulate in the early 1930s in order to preserve the law of the conservation of energy in $\beta$ decay. $^{2}$ This is one of the most celebrated anecdotes in the history of modern physics and introduced the crucial step that fostered the research in neutrino physics, one of the most exciting and promising branches of modern particle physics. Soon after 1956, neutrinos showed themselves to be surprising particles: even if they were supposed to be massless, they immediately attracted the attention of physicists like Bruno

\footnotetext{
E-mail address: silvia.debianchi@uab.cat.

1 On 15 June 1956, Pauli wrote a telegram to Reines and Cowan reporting this quote, see Cowan, Reines, Harrison, Kruse, and McGuire (1956). Tolstoy's quote is taken from War and Peace, Book 10, Chapter 16.

2 In 1930, Pauli wrote his famous letter to the "Radioactive Ladies and Gentlemen" where he sketched out his idea concerning the existence of a new kind of neutral particle. Pauli presented his hypothesis to the scientific community in Pasadena and in Rome in 1931. During the conference in Rome, Enrico Fermi endorsed Pauli's idea in order to develop a theory of $\beta$-decay and also coined the term "neutrino". Pauli presented again to the public the postulate of neutrino in October 1933 at the seventh Solvay Conference in Brussels. For a detailed chronology of the neutrino hypothesis, see Enz (2002). Various versions of this chronology are contained in the manuscripts of Pauli's neutrino lecture (1958) stored in the Pauli Archive at CERN, see also Pauli (1961).
}

Pontecorvo, who in 1957 suggested the neutrino-anti-neutrino oscillation as an explanation for the solar neutrino problem. ${ }^{3}$ In the 1950s and 1960s, theoretical and experimental physicists systematically investigated whether neutrinos possess mass and whether they differ from their antiparticles. The physics of lepton charge and flavor led to a fundamental improvement of the understanding of these particles. Finally, they have been observed to oscillate by the Super-Kamiokande team in 1998, thereby providing evidence of non-zero neutrino mass and of the fact that there are three discrete neutrino masses with different tiny values and not corresponding uniquely to the three flavors. We know that these fermions are subject to flavor mixing and weakly interact with matter. These "ghostly particles" are everywhere, ejected at interstellar distances by exploding supernovas, as observed by Kamiokande in $1987 .{ }^{4}$ Our Sun, for instance, is one of the closest major sources of neutrinos and even our planet produces the so-called "geo-neutrinos". Yet we do not know whether neutrinos can be their own antiparticles, as Ettore Majorana (1937) claimed, but one thing is now clear, namely neutrino physics suggests that there is an

\footnotetext{
3 In 1957, Pontecorvo proposed the hypothesis that neutrinos might "oscillate" and change from one type to another, e.g. from muon to electron neutrino (see Pontecorvo $1957 ; 1963 ; 1983)$. The discovery of neutrino oscillations and muon neutrino mass by the Super-Kamiokande experiment (1998) in Japan confirmed Pontecorvo's idea. In 2015 T. Kajita and A. McDonald received the Nobel Prize for this discovery.

${ }^{4}$ The Kamiokande and IMB experiments detected bursts of neutrinos from SN1987A.
} 
unexplored field that will change our way of understanding many processes in the universe.

This paper aims at showing the relevance of Hermann Weyl's work for the field of neutrino physics, and for the methodological reflections that surround its genesis and development. Since the very beginning, indeed, neutrino physics had been indissolubly linked with the theory of $\beta$-decay and the quantum theory of the electron and positron. ${ }^{5}$ In 1957 the discovery of parity violation in $\beta$-decay not only led to the rehabilitation of Weyl's theory in weak interaction, but also to the revival of Majorana's 1937 theory of the neutrino. The literature acknowledges this fact and presents it as a felicitous example of how a theory developed for completely different purposes suddenly turned out to be useful in interpreting experimental results and in reflecting upon fundamental laws governing interactions (Yang 1986, 12).

The case of parity nonconservation attracted the attention of both historians and philosophers of science (e.g. Pickering 1990, Hoefer 2000, French \& Rickles 2003, Pooley, 2003) for a number of reasons. Among them, there is the fact that it enables a thorough discussion of symmetries in physics and that it represented an interesting case in which experiments challenged fundamental convictions of many physicists. The case of parity violation in $\beta$ decay is considered to be an intriguing one also because it confirmed a possibility entailed in Weyl's two-component theory that was initially criticized by prominent scientists, such as Wolfgang Pauli. However, the process leading to the discovery of parity nonconservation and to the use of the two-component theory of the neutrino is complex and in order to explain how and why Weyl's and Majorana's theories were approached in 1957 one has to consider the history of both theoretical and experimental physics. It is only by bringing them together that an enriched picture of the history of neutrino physics can emerge. Thus, on the one hand, the paper deals with the two-component theory developed by Weyl in 1929 in the context of the study of different theories of spinors. On the other hand, it also takes into account the experiments that led to the discovery of parity nonconservation in order to understand the origin of the revival of Weyl's and Majorana's theories in the 1950s.

As Scholz (2006) underlined, this history is complex and has been only briefly touched by Pais (1986). At present there is a lack of an account connecting Weyl's and Majorana's theories with the history of parity nonconservation in $\beta$-decay that takes into account both experiments and theoretical developments and therefore derives a moral concerning the subtle interrelation among physics and mathematics in the genesis of neutrino physics. Pontecorvo, for instance, considered Weyl (1929b) a milestone in the history of neutrino physics with regard to the consequences it had for the theoretical and experimental understanding of the "elusive particle" and the construction of different types of fermions. In what follows, I shall reconstruct the crucial passages leading to the formulation of Weyl's two-component theory and of how his theory was used in neutrino physics. Section 2 is devoted to explore the fundamental achievements of Weyl's Elektron und Gravitation. In Section 3, I shall analyze the factors leading Weyl to deepen his research on spinors in the 1930s and in Section 4, I shall show how his work influenced Majorana's 1937 theory of electron and positron. In Section 5, I show the role played by Majorana in inspiring Case's formulation of parity violation for massive neutrinos in 1957. In doing so, this paper clarifies the reference made by Pontecorvo in the 1960s to the relevance of Weyl and Majorana for the foundations of neutrino physics and highlights which aspects the field owes to Weyl's research.

\section{Spinors: from mathematics to physics}

Today we are accustomed to talk about Dirac, Weyl and Majorana spinors in describing fermions. In relativistic physics, the classification of spinors is based on the irreducible representations of the Lorentz group $\mathrm{SO}_{+}(1,3)$, even if in general there is no unique definition of spinors, precisely because the research has been developed in the two distinct fields of mathematics and physics. ${ }^{6}$ In order to be oriented in what they called the "Babel of spinors", Vaz \& Da Rocha (2016, pp. 145ff.) proposed a classification of spinors' definitions as (1) classical, (2) algebraic and (3) operatorial. In what follows, I shall concentrate on those classical spinors that were used in physics and on their history dating back to 1926, when Pauli introduced them to describe the spin of the electron nonrelativistically, even if spinors as mathematical entities appeared well before then. It is worth pointing out that in the relativistic case, which is the most interesting one for the purpose of the present paper, spacetime rotations are described by elements of the Lorentz group $S_{+}(1,3)$ and that the groupSL $(2, \mathbb{C})$ is the double covering of theSO $O_{+}(1,3)$. Thus the isomorphism $S L(2, \mathbb{C}) \simeq \operatorname{Spin}_{+}(1,3)$ and the representation space of $S L(2, \mathbb{C})$ is $\mathbb{C}^{2}$, which is the representation space of the group $\mathrm{SU}(2)$ that in turn is the double covering of the special orthogonal group $\mathrm{SO}(3)$. In the relativistic case, the situation is quite different from the nonrelativistic one. In the relativistic case one finds two representations of $S L(2, \mathbb{C})$ that are not equivalent by defining $\rho(A)$ and $\bar{\rho}(A)$ for $A \in S L(2, \mathbb{C})$ as $\rho(A)(z)=A z$ and $\bar{\rho}(A)(z)=\bar{A} z$, where $\bar{A}$ is the complex conjugate matrix associated with $A$,and $z \in \mathbb{C}^{2}$. These two representations are not equivalent because there is no invertible $2 \times 2$ matrix $T$ such that $\bar{\rho}(A) T=$ $T \rho(A) . \bar{\rho}(A) T=T \rho(A)$ does not have solution for $A \in S L(2, \mathbb{C})$, but it has a solution for $A \in S U(2)$. Hence, there are two inequivalent representations of $S L(2, \mathbb{C})$ denoted by $D^{(1 / 2,0)}$ and $D^{(0,1 / 2)}$.

Weyl spinors are those elements of a space that carries each one of such representations and are objects that carry an irreducible representation of the spin group, which is the double covering of the orthogonal group and therefore the spin $1 / 2$ representation of the group of rotations in a quadratic space. Indeed, Dirac spinors are elements of $\mathbb{C}^{4}$ and carry a reducible representation of $\operatorname{Spin}_{+}(1,3) \simeq S L(2, \mathbb{C})$ also represented as the sum of two Weyl spinors, each one corresponding to one of the irreducible representations $D^{(1 / 2,0)}$ and $D^{(0,1 / 2)}$ (see Vaz \& Da Rocha 2016,146). In addition to Weyl and Dirac spinors, it is worth mentioning also Majorana spinors (their application will be discussed in Section 4). These spinors can acquire different physical meanings in particle physics:

1) Dirac four-components theory represents spinors to describe particle and antiparticle,

2) Weyl two-component theory of spinors describes massless particles,

3) Majorana theory represents spinors to describe $1 / 2$ spin particles as their own antiparticle.

The three types of spinors are extremely relevant for particle physics and for the field of neutrino physics in particular. They clearly evolved through time in parallel with the advancement of nuclear physics and quantum field theory. However, the three above-mentioned characteristics and spinor descriptions were present already in the 1930s, before a theory of fermions and bosons was effectively advanced in the 1940s. Moreover, already in the 1930s these three types of spinors had different physical

\footnotetext{
${ }^{5}$ For historical reconstructions of the development of Dirac theory and quantum electrodynamics, see Darrigol 1988; Kragh 1990; Schweber 1994; Roqué 1997; Pashby 2012
}

\footnotetext{
6 The word "spinor" appears in print, for the first time, in van der Waerden (1929), who in turn attributed it to Paul Ehrenfest.
} 
implications that only particle physics experiments could test throughout the second half of the $20^{\text {th }}$ century.

Now, the genesis of Weyl's and Dirac's theories can be analyzed from various perspectives: some preferred to frame them within the development of the debate on unified field theory (Gönner 2004; Goldstein and Ritter 2003), whereas others privileged the history of experimental physics and the search for a practical application of spinors (Pais 1986). In this paper, I will offer a perspective that integrates both these approaches in order to frame Weyl's 1929 theory. In particular, this will allow us to connect the work done by theoretical physicists with the experimental ones and therefore to explain why Majorana theory of spinors arose in the mid-1930s within the context of Fermi's group in Rome that devoted most of its effort to the experimental work on neutral particles and $\beta$-decay. This approach integrating the history of theoretical and experimental physics leads us to reflect upon the interrelations of theoretical and experimental physics with mathematics, thereby showing that the possibility of having different types of spinors (and therefore our capacity to describe different fermions) was not simply due to the desire to accommodate experimental results with theory. The study of alternative theories of spinors, indeed, is also the result of an effort made by mathematical physicists to develop possibilities contained a priori in the investigations of the properties of the Lorentz group. This approach was fully appreciated in the late 1950s and 1960s, when the role of groups in the foundations of particle physics had shown its power of unification and prediction of phenomena.

\subsection{Framing Weyl's two-component theory}

A number of works (Pais 1986; Straumann 1997; Straumann 2001; Scholz 2005) framed Weyl's two-component theory within the development of the debate on unified field theory and the application of spinors to atomic and nuclear physics. In particular, Scholz (2005) underscored how Weyl's 1929 theory mirrored a conceptual change in his research programme with respect to the early 1920s, namely Weyl took experimental physics to be more and more important in informing his proposal of a "geometry of matter" and aimed at breaking with the prevailing tendency of geometrization in unified field theory. In this section, I shall briefly consider Pauli's work, in order to show its relevance for Weyl's criticism of distant parallelism (see also Section 2.2), as well as to emphasize conceptual aspects of Weyl's theory that Pauli criticized, but that were also crucial in informing Weyl's theory of spinors in the 1930s (see Sections 2.3 and 3).

When Uhlenbeck and Goudsmit (1925) postulated the existence of an intrinsic angular momentum for the electron in order to provide an explanation of the Stern-Gerlach experiment, there was no solid theoretical framework for their postulate. The latter was obtained through the works of Pauli (1927), in the non-relativistic regime, and Dirac (1928a; 1928b), in the relativistic one. ${ }^{7}$ The development of spinor theory in modern physics thus started as a need to provide a framework to experimental results. In particular, the observation of the spin property of elementary particles required new theoretical investigations into 1) the nature of Fermi statistics and 2) the description of these objects by suitable wave functions. ${ }^{8}$ Pauli did not react positively towards the proposal of Uhlenbeck and Goudsmit (1925). In particular, he did not believe that the mechanical picture

\footnotetext{
${ }^{7}$ Dirac was able to show that the properties of electron spin agreed with Uhlenbeck and Goudsmit's postulate and the results of the Stern-Gerlach experiment (see Mehra \& Rechenberg 2001, 1032-1033).

${ }^{8}$ As to the second question, Darwin (1927) marked an important contribution when he represented the electron by a vector wave instead of a scalar wave.
}

of a rotating electron provided an adequate allowed description of his fourth quantum number, because such an eigenrotation would imply unacceptable consequences for the structure of the electron. Furthermore, in 1926 Pauli noticed that Heisenberg and Jordan's theory treated intrinsic spin as a traditional vector quantity, but it was clear from the hydrogen spectrum that such a treatment was insufficient. Thus, Pauli developed his own theory in which spin was given by a classically indescribable two-valuedness.

In a letter to Jordan dated 12 March 1927, Pauli finally claimed that he had shown why spin can assume only two positions in a magnetic field (see Pauli 1979, 385-386). This crucial point together with other results of his investigation were collected in Zur Quantunmechanik des magnetischen Elektrons published in Zeitschrift für Physik in late 1927. This work contains Pauli's two-component theory. ${ }^{9}$ At the beginning of the paper, Pauli stressed the fact that in contrast with the situation of matrix mechanics, the introduction of the electron spin into matrix mechanics generated formal difficulties arising from the double-valuedness of the eigenfunctions. Darwin (1927) also emphasized this point. However, instead of choosing Darwin's approach, Pauli proposed a simpler method to solve the problem, namely he added "to the position variables of the center-of-mass of the electron the components of the spin of each electron in a fixed direction (instead of the rotating angle conjugate to the spin variable)" (Pauli 1927, 603)..$^{10}$ Nevertheless, Pauli's theory had two main problems. First, it was non-relativistic and, therefore, was only approximately true for low velocities. Second, and perhaps even more importantly, it was unable to predict a factor of 2 in the gyromagnetic ratio. Despite these major problems, Pauli (1927) was an important source of inspiration for Weyl's 1929 work (see Weyl 1929a, 330-331) for reasons that involve the debate on unified field theory. In between 1927 and 1929, Wiener and Struik (1927) and Zaycoff (1929) among others, tried to provide a geometrical framework to Schrödinger's, Dirac's or the Klein-Gordon equation by connecting the quantum mechanical wave function to geometrical objects in order to unify gravitation and electricity to quantum theory. ${ }^{11}$ In 1929 Weyl entered the debate by highlighting how "it is now hopeless to seek a unification of gravitation and electricity without taking material waves into account" (Weyl 1929a, 325). Weyl thus contributed to these attempts by emphasizing that elements of spin space, i.e. spinors, were to be used in order to represent the electrons in the form of Dirac's equation. ${ }^{12}$ As the simple representation of the Lorentz group, spinors and their use became widely studied by the proponents of unified field theory and discussed from different perspectives by Einstein (1928), and later by Schouten (1931) and Einstein and Mayer (1931). ${ }^{13}$ Fock (1929), for instance, emphasized the connection between Einstein's 1928 theory of teleparallelism and the spin theory of the electron. ${ }^{14}$ Precisely in contrast to this

\footnotetext{
${ }^{9}$ In the non-relativistic case the group $\mathrm{SU}(2)$ is isomorphic to the group $\mathrm{Spin}(3)=$ $\operatorname{Spin}(3,0)$. From the isomorphism $\mathrm{SU}(2) \simeq \operatorname{Spin}(3)$, one concludes that $\mathbb{C}^{2}$ is the representation space of the group Spin(3). Pauli spinor is an element of the representation space of the group Spin(3), which is the spin $1 / 2$ representation of the group of three-dimensional spatial rotations.

10 The original German text reads: “Dies gelingt nämlich dadurch, daß man zu den Lagenkoordinaten $q$ der Elektronenschwerpunkte die Komponenten des Eigenimpulses jedes Elektrons in einer festen Richtung (statt der zu diesen konjugierten Drehwinkel) als neue unabhängige Variable hinzufügt".

11 For the development of these approaches, see Jehle (1934; 1935)

12 The problem was to combine spinors with the vectors and tensors appearing in electromagnetism and gravitational theory (see Weyl 1929b, 331-332).

13 For a detailed reconstruction, see Gönner (2004), 100ff.

14 Fock (1929), for instance, proposed to introduce the use of spinors under the assumption of teleparallelism proposed by Einstein (1928). For detailed discussion, see Barbour and Vizgin (2011). For the development of Einstein (1928), see Einstein and Mayer (1931).
} 
approach, Weyl, like Pauli, distinguished his theory, by dispensing with teleparallelism.

From 1928 onward, like most of his colleagues, ${ }^{15}$ Weyl worked on Dirac's theory and recognized the great achievement of Dirac (1928a, 1928b) in the first edition of Gruppentheorie und Quantenmechanik (1928) ${ }^{16}$ However, differently from other physicists, such as Pauli and Heisenberg, who were mostly worried about the predicted existence of positive-energy levels and of an equal number of negative-energy levels (see Kragh 1981, 55; 63 ff.), Weyl concentrated his efforts in the incorporation of the two-component theory of spinors within gravitational theory. This preoccupation led him to search for a new formulation of gauge invariance and to work on the theory of spinors from 1929 onward.

\subsection{Weyl two-component theory}

In April 1929, Weyl published the first version of his Gravitation and Electron in the Proceedings of the National Academy of Sciences (see Weyl 1929a). Pauli criticized this version of Weyl's paper, but he changed his mind after reading the second German version and writes to Weyl:

In contrast to the nasty things I said, the essential part of my last letter has since been overtaken, particularly by your paper in $\mathrm{Z}$ [eitschift]. f[ür]. Physik. For this reason, I have afterward even regretted that I wrote to you. After studying your paper I believe that I have really understood what you wanted to do (this was not the case in respect of the little note in the Proc[eedings of the]. Nat[ional]. Acad[emy].). First let me emphasize that side of the matter concerning which I am in full agreement with you: your incorporation of spinor theory into gravitational theory. I am as dissatisfied as you are with distant parallelism and your proposal to let the tetrads rotate independently at different space-points is a true solution (Pauli 1979, 518).

A second extended German version entitled Elektron und Gravitation appeared in Zeitschrift für Physik in 1929 and represented one of the fundamental contributions for the development of gauge theory (see Straumann 1997; O'Raifeartaigh \& Straumann, 2000) and the modern concept of gauge invariance:

The Dirac field equations for $\psi$ together with the Maxwell equations for the four potentials $f_{p}$ of the electromagnetic field have an invariance property which is formally similar to the one which I called 'gauge invariance' in my 1918 theory of gravitation and electromagnetism; the equations remain invariant when one makes the simultaneous substitutions

$\psi$ by $e^{i \lambda} \psi$ and $f_{p}$ by $f_{p}-\frac{\partial \lambda}{\partial x^{p}}$

where $\lambda$ is understood to be an arbitrary function of position in four-space. Here the factor $\frac{e}{c h}$ where $-e$ is the charge of the electron, $c$ is the speed of light and $\frac{h}{2 \pi}$ is the quantum of action has been

\footnotetext{
15 Not only Pauli, but also Heisenberg expressed dissatisfaction with Dirac's theory, e.g. in a letter to Pauli in May 1928 (Pauli 1979, 443) or July 1928 (Pauli 1979, 467).

${ }^{16}$ Weyl's work also played an important role in leading Dirac to clarify his own theory of the spinning electron (see Weyl 1929a, 332; Kragh 1981, 63 ff.). According to Kragh $(1981 ; 1990)$, nevertheless, the notion of negative energy state did not represent a problem for Dirac, at least immediately. His theory was driven by mathematical and practical considerations and he was far more interested in developing the potential of his formalism, rather than in adjusting and refining the conceptual meaning of the latter in view of a coherent theory.
}

absorbed in $f_{p}$. The connection with this "gauge invariance" to the conservation of electric charge remains untouched. But a fundamental difference which is important to obtain agreement with observation is that the exponent of the factor multiplying $\psi$ is not real but imaginary. $\psi$ now plays the role that Einstein's $d s$ played before. It seems to me that this new principle of gauge invariance, which follows not from speculation but from experiment tells us that the electromagnetic field is a necessary accompanying phenomenon, not of gravitation, but of the material wave field represented by $\psi$. Since gauge invariance involves an arbitrary function $\lambda$ it has the character of "general" relativity and can naturally only be understood in this context. (Weyl 1929b, 331)

In 1929 Weyl's new concept of gauge invariance aimed at unifying material wave field and the electromagnetic field in such a way to express constancy under simultaneous transformation of the wave field $\psi$, obeying the Dirac equation for a spinor of rest mass mand charge $e$ in Minkowski space,

$\left[i \gamma^{k}\left(\partial_{k}-i e A_{k}\right)-m\right] \psi=0$

and of the electromagnetic four-vector potential $A_{i}$ contained therein, according to

$\psi \rightarrow \psi^{\prime}=e^{i \theta} \psi, A_{i} \rightarrow A \prime_{i}=A_{i}+\frac{1}{e} \frac{\partial \theta}{\partial x^{i}}$

where $\theta\left(x^{i}\right)$ is an arbitrary real function of the space-time coordinates $x^{i}$ (see Weyl 1929b, 331). The exponent in the factor multiplying $\psi$ in (2) is purely imaginary (see Weyl 1929b, 331), and Weyl also emphasized the general relativistic nature of the transformations (2) (see Weyl 1929b, 331). Weyl therefore started investigating more in depth Dirac theory (Weyl 1929b, 330), in which $\psi$ is represented as a four-component spinor that results in a doubling of the number of energy levels (cf. Dirac 1928a, 1928b; see Weyl 1929b, 331). Weyl argued that, without giving up relativistic invariance, one should return to Pauli (1927) two-component theory in order to describe massless spinors $\psi \equiv\left(\begin{array}{l}\psi_{1} \\ \psi_{2}\end{array}\right)$. However, in Dirac theory the two two-component spinors $\psi_{1}$ and $\psi_{2}$ become linked together if the spinor is massive, because of the presence of off-diagonal elements in the Dirac gamma matrices, which occur in the kinetic term $i \gamma^{k} \partial_{k} \psi$, but not in the mass term $m \psi$. In Weyl representation, thus we have

$\gamma_{0}=\left(\begin{array}{cc}0 & -\sigma_{0} \\ -\sigma_{0} & 0\end{array}\right), \gamma_{\alpha}=\left(\begin{array}{cc}0 & \sigma_{\alpha} \\ -\sigma_{\alpha} & 0\end{array}\right)$

where the generalized $2 \times 2$ Pauli matrices $\sigma_{i}$ are defined by

$\sigma_{0}=\mathbf{1}=\left(\begin{array}{ll}1 & 0 \\ 0 & 1\end{array}\right), \sigma_{1}=\left(\begin{array}{ll}0 & 1 \\ 1 & 0\end{array}\right), \sigma_{2}=\left(\begin{array}{cc}0 & -i \\ i & 0\end{array}\right), \sigma_{3}=\left(\begin{array}{cc}1 & 0 \\ 0 & -1\end{array}\right)$

As a result, (1) reduces to the two-component equations

$i\left[\left(\partial_{0}-i e A_{0}\right)-\sigma^{\alpha}\left(\partial \alpha-i e A_{\alpha}\right)\right] \psi_{2}+m \psi_{1}=0$

and

$i\left[\left(\partial_{0}-i e A_{0}\right)+\sigma^{\alpha}\left(\partial \alpha-i e A_{\alpha}\right)\right] \psi_{1}+m \psi_{2}=0$.

The spinors $\psi_{1}$ and $\psi_{2}$ only decouple if $m=0$. Furthermore, Eqs. (5) and (6) can be reduced to

$i\left(\partial_{0}-\sigma^{\alpha} \partial_{\alpha}\right) \psi_{2}=0$

and 
$i\left(\partial_{0}+\sigma^{\alpha} \partial_{\alpha}\right) \psi_{1}=0$

respectively, and it is immediately clear that the difference by a sign in the second term breaks the symmetry between $\psi_{1}$ and $\psi_{2}$. In physical terms, as remarked by Weyl (1929b, 332), this means to abandon the left-right symmetry, so that under projection the leftand right-handed components read as

$\psi_{L}=\frac{1}{2}\left(1+\gamma_{5}\right) \psi=\psi_{1}, \psi_{R}=\frac{1}{2}\left(1-\gamma_{5}\right) \psi=\psi_{2}$.

This result led Weyl to express the hope, given the gravitational origin of mass that it would be possible in a new formulation of the Dirac equation to replace $m$ by a geometrical term (see Weyl 1929b, 331). Indeed, as Weyl noticed:

"The theory of Dirac in which the wave field of the electron is described by a potential with four components and provides twice the amount of energy levels; hence one should be able to return, without renouncing to relativistic invariance, to Pauli's two-component theory. One is prevented from doing this by the term in Dirac's equation containing the mass as a factor" (Weyl 1929b, 330-331).

Dirac's four-component wave function, indeed, splits into two pairs and Weyl believed that in a satisfactory theory it must have been impossible to introduce a non-vanishing mass without the gravitational field "as it is to introduce charge without the electromagnetic field" (see Weyl 1929a, 323). Independently of Weyl, Fock (1929) also incorporated the Dirac equation into general relativity using a two-component theory, but, contrary to Weyl, he did not dismiss Einstein's distant parallelism at that time. ${ }^{17}$ Weyl's twocomponent theory, instead, had the advantage of only "simulating" Einstein's teleparallelism ("Immerhin wäre hier der Fernparallelismus nur vorgetäuscht”, see Weyl 1929b, 352), but it also had the disadvantage of leading to the possibility of parity nonconservation. An option that could not be accepted then by the scientific community for reasons that will be discussed in the next sections. Weyl 1929 two-component theory, therefore, represented an alternative to approaches, such as Einstein's and Fock's, but also a fundamental attempt at using Pauli two-component theory in a relativistic framework. As a matter of fact, indeed, Weyl's approach heavily inspired later works, such as Infeld and van der Waerden's (1933) that described two-component spinor fields without appealing to distant parallelism. Even if Pauli converged with this aim and recognized the great value of Weyl's new formulation of the concept of gauge invariance, yet he could not endorse Weyl's view completely, and the reason was that Weyl's two-component theory implied parity nonconservation.

\section{Weyl's view of parity}

In his 1929 paper, Weyl pointed out that the $\operatorname{SL}(2, C)$ transformation excludes time-reversal $T$ and considered this a positive aspect of his theory because it took into account the fact that nature is not time-reversible. However, Weyl two-component theory also excludes the linear implementation of parity (see Straumann 2001; Coleman \& Korté 2001). But what was Weyl's view of parity emerging from his two-components theory? Pauli noticed in the 1933 Handbuch der Physik that Weyl's theory raised a more serious problem than Dirac's notion of negative-energy states or arising from the electron

\footnotetext{
17 See Fock and Iwanenko (1929). For a comparative study of Weyl's and Fock's 1929 works on spinors, see Scholz (2005).
}

and positron theory (Pauli 1933, 85). Weyl himself emphasized that, since the two-component theory excluded a linear implementation of parity, "it is only the fact that the left-right symmetry appears in Nature that forces us to introduce a second pair of $\psi$ components" (Weyl 1929b, 334). The literature offers two readings of this statement. Notably, Yang $(1986,12)$ claimed that similarly to Pauli, also Weyl did not accept parity violation. Enz (2002) rather emphasized that by reducing the Dirac $\psi$ from four to two components, the negative-energy states could have been avoided and Weyl considered parity violation to be a small price to pay for his two-component theory. Pauli actually believed that the wave equations of Weyl's theory were "not applicable to physical reality" (Pauli 1933, 234), ${ }^{18}$ because they were not invariant with respect to reflections, i.e. exchange of left and right. However, as Straumann $(2001,148)$ remarked, Weyl's wave equations are both invariant under the exchange of left and right when interactions are not taken into account. Nevertheless, even if Pauli admitted the relevance of Weyl's new formulation of the principle of gauge invariance for physics, yet the implication of parity nonconservation in the two-components profoundly disturbed his convictions. It must be recalled that Pauli had good reasons to criticize Weyl about this point. According to Laporte's selection rule, indeed, the transitions in the energy levels of a spectrum of iron always occurs from "gestrichene" to "ungestrichene" levels or vice versa (Laporte 1924), corresponding in current terms to even and odd levels. A convincing explanation of the existence of two types of levels was found in 1927, when Wigner showed that the two types of levels, as well as the selection rule derived from the invariance of the Schrödinger equation under the operation of inversion of coordinates $x \rightarrow-x, y \rightarrow-y, z \rightarrow-z$ (see Wigner 1927). The term "Spiegelung" was then indissolubly linked to the property of parity conservation ${ }^{19}$ and this was not only quickly accepted, but also became a "sacred cow" for the physics community (Wroblewski 2008). If we consider Weyl's approach, however, we see that differently from Pauli, he assumed the need of using Dirac four-component theory on the ground of the lack of experimental observation of parity nonconservation in interactions. Straumann $(1997,110)$ rightly pointed out that to Weyl the mass problem of Dirac theory would eventually be solved by the theory of gravitation and that he attributed the need for the use of Dirac four-component theory to parity rather than to mass (see Weyl 1929b, 331). However, in my view, Weyl did not treat parity as a "sacred cow". He rather assumed the need of a four-component theory because in nature there was no experimental evidence for parity nonconservation. ${ }^{20}$ This fact gives to parity nonconservation in Weyl's two-component theory the status of a possibility to be developed mathematically that cannot be said to be false or impossible, because there is no contradiction in making the left-right symmetry disappear: "we shall see that two components are sufficient, if the symmetry of left and right is suppressed" (Weyl 1929, 332). ${ }^{21}$

As we shall see in the next sections, Weyl's approach to parity conservation is extremely significant, given that his twocomponent theory will constitute the case study used by Lee and Yang (1957) to support the discovery of parity nonconservation and the implementation of the theory of weak interactions. Furthermore, as show in the next section, this aspect is also

\footnotetext{
18 The conclusion concerning the non-applicability to the physical reality is cancelled in the 1958 edition of Pauli (1933), see Enz 2002, 254-255.

19 At least until 1933, since the term was still used by Pauli. The name "parity" appeared later, when Condon and Shortley (1935) used the term "parity operator".

20 Cf. Weyl 1929, 334: "Nur diese tatsächlich in der Natur bestehende Symmetrie von rechts und links wird uns zwingen, ein zweites Paar von $\psi$-Komponenten einzuführen".

21 Translation is mine. The original text reads: "Wir werden sehen, daß man mit zwei Komponenten auskommt, wenn die Symmetrie von links und rechts aufgehoben wird".
} 
relevant to understand Majorana's 1937 formulation of the theory of neutrino. But before dealing with this, I further substantiate my claim about Weyl's view of parity by considering important aspects of the development of his research on spinors in the 1930s. It is worth noticing, indeed, that Weyl did not stop his research on spinors after 1929 when he was visiting Princeton. Weyl continued his work on spinors when moved back to Princeton in 1934 as member of the Institute for Advanced Study (IAS). Also thanks to the research done by Oswald Veblen and his collaborators in the Mathematics Department and later in the Mathematics School of the IAS, Weyl found a vibrant environment that stimulated and encouraged his research (see Scholz 2001; Ritter 2011). Veblen established the connection between spinors and projective geometry and developed its consequences in lectures that he gave jointly with Givens. Veblen's works on spinors (Veblen 1933a, 1933b, 1933c) inspired Weyl's work in the 1930s, but in turn Veblen himself considered his 1933 Geometry of two-component spinors "a sort of geometric commentary on the paper of Weyl" (Veblen 1933b; Gönner 2004, 103). Of Course Veblen had no immediate interest in showing a connection between spinors and physics and rather sought to contribute to the development of projective geometry. However, he created a fertile environment for receiving and discussing Weyl's research, especially in Princeton. In 1935, Brauer and Weyl gave a general construction of the spin representations in any number of dimensions and showed how the tensor product of two such representations can be decomposed into irreducibles. In order to understand how Weyl was led to this result, one should consider both the optimal environment of the research pursued in Princeton, as well as the opportunity he had to work with other excellent mathematicians, such as Richard Brauer, who pursued an algebraic approach to spinors. Indeed, with the advent of the Nazi regime in Germany, among the several academics and refugees escaped from Europe there was Brauer, who was fired in 1933 at the University of Königsberg. Thus, Brauer was offered a short-term appointment in Lexington, Kentucky, in the academic year 1933-34 and in the following year, he became Weyl's assistant at the IAS. Weyl's interest in Brauer's work and their mutual appreciation led to a collaboration on many projects, whose most important result was the famous joint paper on spinors published in the American Journal of Mathematics in 1935. Brauer and Weyl (1935) found global, spinorial representations of the groups $\operatorname{Spin}(n)$ for all $n$. This road to spinors is topological and it is related in an essential way to the non-triviality of the fundamental groups $\pi_{1}$ of the groups of rotations. ${ }^{22}$ This means that such an approach allows a generalization of the notion of spinor representations to the general linear groups, namely spinors associated with the general linear group have an infinity of components (see Ne'eman 1978; Budinich and Trautman, 2012). In constructing spinors in $n$-dimensions, Brauer and Weyl confronted their theory with Dirac's theory of the electron. They claimed that there was a complete agreement with it. However, they also pointed out that the possibilities implied by Dirac's theory were not completely developed if one assumes the equivalence of left and right:

"Let us suppose we are dealing with a spinor field $\psi^{A}\left(x^{1} \ldots x^{n}\right)$ in an $n$-dimensional 'world' with the fundamental metric form (33). ${ }^{23}$ The most essential feature of Dirac's theory is that one should be able to form a vector by linear combination of the products $\bar{\psi}^{A} \psi^{B}$. If $n$ is even, one sees from equation (40) that exactly one such vector $s_{i}$ exists-that behaves like a vector at

\footnotetext{
22 The topological approach to spinors is more general that the one based on the idea of linearization of a quadratic form.

${ }^{23}$ Here Brauer and Weyl refer to the real normal form: $-\left(x^{1}\right)^{2}-\ldots-\left(x^{t}\right)^{2}+$ $\left(x^{t+1}\right)^{2}+\ldots+\left(x^{n}\right)^{2}=\sum_{i} \epsilon_{i}\left(x^{i}\right)^{2}$, supposing $2 t \leq n$.
}

least for all Lorentz transformations not reversing the sense of time; and one such vector for all Lorentz transformations not reversing the spatial sense. In the case $n$ odd, one vector of the second, and no vector of the first kind exists. Only the first type can be used when one believes in the equivalence of right and left, but is prepared to abandon the equivalence of past and future" (Brauer and Weyl 1935, 446-447).

Among other possibilities to be developed there was the nonconservation of the equivalence of left and right, and therefore a parity nonconservation depending on cases in which $n$ is even or odd. ${ }^{24}$ This passage clarifies Weyl's view of parity nonconservation. On the ground of mathematical considerations, Weyl did not reject the possibility of parity nonconservation, even if physically nobody could provide any experimental evidence in its favor. In this sense, the history of spinors provides a good example of the subtle interrelations and mutual influences between mathematics and physics. Veblen in Spinors and Projective Geometry, for instance, remarked that.

"The theory of spinors like so many other branches of mathematics received its first impulse from theoretical physics. Unlike some other branches it has not required the discovery of anything very fundamentally new on the mathematical side, but rather has called for a regrouping and restudy of new ideas" (Veblen 1937, p. 111).

The fact that in 1957 Weyl's theory was able to provide a theory of massless neutrinos including parity violation is a milestone that can be understood only by thinking of the complex relationship between mathematics and physics (both theoretical and experimental physics) and by considering the historical development and dissemination of Weyl's theory in the 1930s and 1940s. Veblen's claim that spinors did not required any exceptional mathematical discovery is certainly true. However, their use and the need of applying them to read the book of nature led physicists to make mathematical discoveries, as it happened in the case of Majorana.

\section{Weyl's echoes: the case of Majorana's symmetric theory of electron and positron (1937)}

In the 1930s Weyl continued working on spinors while in Princeton and other prominent figures in Europe where pursuing this research from different perspectives. Pauli hypothesis of the existence of neutrino, Fermi's theory of $\beta$-decay and the discovery of the positron inspired and profoundly shaped the research in both theoretical and experimental nuclear physics in Europe. It is now my intention to briefly discuss how Weyl's work was considered in Fermi's group in Rome in the 1930s and, in particular, I emphasize its role in informing Ettore Majorana's work. ${ }^{25}$ At the beginning of the 1930s, Fermi's group paid specific attention to experiments on nuclear physics and from 1934 onwards to testing his theory of $\beta$ decay that assumed the existence of the particle postulated by Pauli, called by Fermi "neutrino" (see Fermi 1934). Only a few

\footnotetext{
24 In various works (Weyl 1949, p. 208, Weyl 1931, Weyl 1948, Weyl 1931b, 147; 172) Weyl explicitly discussed the question of the interchange of left and right and clarified in which sense this interchange is not a problem per se, even if it opens fundamental questions concerning the constitution of matter in our universe.

${ }^{25}$ For Majorana's biography, see Amaldi (1966), who reports that in the early 1930s Majorana considered Weyl's book the best and deeper book on quantum mechanics.
} 
members investigated Fermi's theory of $\beta$-decay and the development of suitable descriptions of spin via wave functions. ${ }^{26}$ Among them, there were Giovanni Gentile jr. and Ettore Majorana, who were extremely interested in studying and developing a group theoretic approach to quantum mechanics (Bonolis 2004). Their work was heavily influenced by Weyl's 1929 paper and by Gruppentheorie und Quantenmechanik. Precisely the work on unitary representations of the inhomogeneous Lorentz group was a central preoccupation of Majorana in 1930-1931 as the publication of his Relativistic Theory of Particles with Arbitrary Intrinsic Angular Momentum (1932) testifies. In this paper, he tried to develop a relativistically invariant linear theory for particles with arbitrary spin, both integer and semi-integer, in which all mass eigenvalues are positive. Majorana (1932) remained almost unnoticed until the late-1930s when Pauli and Fierz discussed it in their correspondence, $^{27}$ and Wigner reported that "the representations of the Lorentz group have been investigated repeatedly. The first investigation is due to Majorana, who in fact found all representations of the class to be dealt with in the present work excepting two sets of representations" (Wigner 1939, §2). Nevertheless, the importance of the achievements contained in Majorana (1932) were recognized by van der Waerden in 1933, as Majorana himself reported in a letter written to his father during his research stay in Leipzig where he was working with Heisenberg:

“In my last paper appeared on 'Nuovo Cimento' is contained an important mathematical discovery, as I could ascertain through a conversation with Prof. van der Waerden, a Dutchman teaching here, one of the leading authorities in group theory" (Majorana, Third Letter from Leipzig, 18 February 1933, in Recami 1991, 125). ${ }^{28}$

What was the mathematical discovery mentioned in the letter? Majorana found that the Klein-Gordon equation, the Dirac equation and Weyl's two-component theory are all related to the appropriate irreducible representations of the Poincaré group and this achievement is mentioned in Wigner (1939). ${ }^{29}$ Furthermore, Majorana's interest in pursuing Weyl's approach to quantum mechanics also emerges in Symmetric theory of the electron and positron (1937). Majorana's aim was to show that it was possible to achieve "a complete formal symmetrization in the electron and positron quantum theory by means of a new quantization process" (Majorana 2006[1937], 218). This could have been achieved by modifying the meaning of Dirac equations in such a way that there was no longer need to deal with negative energy states nor to presume for any type of particles, especially neutral ones, "the

\footnotetext{
${ }^{26}$ According to Bonolis (2004), Majorana's interest in group theory probably arose at the beginning of 1928, thanks to Giovanni Gentile jr, a former pupil of the mathematician Luigi Bianchi. Majorana owned his books, together with the first German edition of Weyl (1928) and Speiser (1923). Fermi owned the 1931 edition of Weyl's book and discussed it in seminars and lectures but Fermi was not a proponent of the application of group theory to quantum mechanics (See Segrè 1970, 56).

27 Interest for Majorana's work was also expressed by Fierz and Pauli. In 1939 they shared their views about the reality of the Majorana condition (Majorana 1937, 226) and about the implications of considering neutrino as a particle possessing mass and being its own antiparticle, see also Enz (2002, 349-350).

28 Translation is mine. The original text reads: "Nell'ultimo mio articolo apparso sul 'Nuovo Cimento' è contenuta una importante scoperta matematica, come ho potuto accertarmi mediante un colloquio col professor van der Waerden, olandese che insegna qui, una delle maggiori autorità in teoria dei gruppi".

29 The relevance of Majorana (1932) was recognized in the Soviet Union where from 1948 to 1958 appeared several studies devoted to Majorana's infinite component equation, e.g. Gelfand and Yaglom (1948). See also Fradkin (1966) who noticed that matrix elements identical with Majorana's infinite dimensional Lorentz representations were given by Weyl $(1928,160)$.
}

existence of antiparticles corresponding to the 'holes' of negative energy" (see Majorana 2006[1937], 218). Majorana thus raised a fundamental question for elementary particle physics: the question about the true neutrality of electrically neutral spin $1 / 2$ particles. ${ }^{30} \mathrm{~A}$ Dirac particle is one which is distinct from its antiparticle, in contrast Majorana formally introduced in 1937 the concept of a particle which is identical to its antiparticle and this characterization is still present in our current classification of spinors (see Section 2 above). In his 1937 paper, Majorana chose a representation according to which the four gamma matrices are pure imaginary to embody the complete symmetry of the quantized Dirac field theory between particles and antiparticles. Then he suggested to apply it to neutrons and still hypothetical neutrinos. ${ }^{31}$ By explicitly following upon Weyl's group-theoretic approach as developed in Weyl (1928), ${ }^{32}$ Majorana offered a solution that still informs current research in neutrino physics and informed research in supersymmetric theories. In order to suggest that a neutral spin $1 / 2$ particle can be its own antiparticle, ${ }^{33}$ he first proposed to impose a reality condition on Dirac spinor $\psi$, according to which the representation of $\gamma^{\mu}$ matrices has a new basis (Majorana basis):

$$
\begin{aligned}
\gamma^{0} & =\left(\begin{array}{cc}
0 & \sigma^{2} \\
\sigma^{2} & 0
\end{array}\right), \gamma^{1}=\left(\begin{array}{cc}
i \sigma^{3} & 0 \\
0 & i \sigma^{3}
\end{array}\right), \gamma^{2}=\left(\begin{array}{cc}
0 & -\sigma^{2} \\
\sigma^{2} & 0
\end{array}\right), \gamma^{3} \\
& =\left(\begin{array}{cc}
-i \sigma^{1} & 0 \\
0 & -i \sigma^{1}
\end{array}\right)
\end{aligned}
$$

and

$$
\gamma_{5}=\left(\begin{array}{cc}
\sigma^{2} & 0 \\
0 & -\sigma^{2}
\end{array}\right)
$$

The matrices not only satisfy the Clifford algebra, but also are all pure imaginary, so that the generators of the Lorentz group are all real. With this basis of the Clifford algebra, one works with a real spinor simply by imposing the condition, $\psi=\psi^{*}$ which is preserved under Lorentz transformation. Now, the reality conditions imposed to the field quantities also means that Majorana spinors have a twocomponent representation, as it was the case with Weyl's theory. In Majorana theory not only the components are invariant under charge conjugation, but also the particle and antiparticle are indistinguishable as long as the Majorana condition is satisfied. ${ }^{34}$ The aim of Majorana consisted in showing that the prescriptions of Dirac's theory are inconvenient, or better, "not satisfactory". Majorana claimed that it was the case "because one starts with an

\footnotetext{
${ }^{30}$ In an interview Heisenberg declared: "The Dirac spinor was the thing which everybody discussed, but then there was Weyl spinor business which van der Waerden knew. The others did not know about it, but then there was Majorana. He was in Leipzig and Majorana found his Majorana particle, which has no charge but still had the spin $1 / 2$, and that, of course, was to be represented by Weyl's spinor. So we learned a lot from van der Waerden just in that respect." (Interview of Werner Heisenberg by Thomas S. Kuhn and John L. Heilbron on 1963 July 5, Niels Bohr Library \& Archives, American Institute of Physics, College Park, MD USA, www.aip.org/history-programs/niels-bohr-library/oral-histories/4661-11).

31 Furthermore, Majorana's two-component theory of the neutrino was a quantized field theory, while Weyl's 1929 theory appeared in Zeitschrift für Physik was not.

32 For studies on the influence exerted by Weyl on Majorana, see Enz (2002, 350351); Drago and Esposito (2004).

33 Neutrinos definitely have anti-particles, but another question is whether an antiparticle differs from its particle or not.

${ }^{34}$ The Majorana condition is $\psi=\psi^{C}$, which in terms of the four components leads to $\psi L=\psi_{R}^{C}$ and $\psi R=\psi_{L}^{C}$. This can be read in two different ways, depending on whether one addresses $\psi L, \psi_{L}^{C}$ or $\psi L, \psi R$ as independent basic states. In the first case there is a left-handed neutrino and its right-handed antiparticle, whereas in the second case one regards the tuple $(\psi L, \psi R)$ as the left- and right-handed components of a single particle that is identical to its antiparticle (see Giulini 2009).
} 
asymmetric form and because symmetric results are obtained only after one applies appropriate procedures such as the cancellation of divergent constants, that one should possibly avoid" (Majorana 1937, 218-219). In other words, he was following upon Pauli, Heisenberg and Weyl in his critique of Dirac, but the results of Majorana's theory were more radical and admitted parity nonconservation for true neutral particles (Majorana 1937, 219) ${ }^{35}$ and the postulate that neutrinos possess a tiny mass. The construction of Majorana's theory for neutral particles, indeed, postulated a mass term for both neutrons and neutrinos. The reasons for proposing an alternative to Dirac's theory in the case of neutral particles were therefore not simply due to an excessive fanaticism for the formalism. They were rather meant to develop the implications of Weyl's two-component theory (Serpe 1952) in order to frame neutrino physics within Fermi's theory of $\beta$ decay by deriving a theory of neutral particles possessing mass from a priori mathematical grounds (Touschek 1948). ${ }^{36}$ Majorana theory thus can be read along the lines of the perspective encouraged by Weyl himself, according to which mathematics possesses the feature of being open to the realm of infinitely many possibilities that theoretical and experimental physicists determine and explore in order to provide the explanation and prediction of physical phenomena and interactions. In Weyl (1929c), indeed, the mathematical method is characterized as the "a priori construction of the possible" in opposition to the "a posteriori description of what is actually given". Majorana, indeed, followed upon Weyl in endorsing his view of the relationship between physics and mathematics and was able to show that there was a methodology exploiting group theory that allowed on pure mathematical grounds the construction of a symmetric theory of electron and positron devoid of the concept of negative energy states. However, the price to pay in order to obtain such a result was to accept the possibility of parity violation for neutral particles, such as the neutrino, in $\beta$-decay. Such a possibility was unacceptable in the 1930 s and it was widely discussed only after Wu's experiment took place in 1956.

\footnotetext{
35 According to Pontecorvo (1982, 229-230): "It is amazing how much is implied, explicitly, or implicitly, in his [n.a. Majorana's] famous paper. I have already stressed that there one can either see or see between the lines electrically neutral fermions both without any charge and with some charge (lepton, baryon .). True, implicitly all charges are supposed to be strictly conserved, but this is not stated in words. Now we know that among bosons there may be "hybrid particles", that is bosons having a charge which is not strictly conserved and oscillating between two different states like neutral kaons. If there exist such electrically neutral hybrids among fermions, we would expect that they are not described by stationary states; that they oscillate one into another and that they are superpositions of particles with definite, different masses, which are described by stationary states and are truly neutral (or Majorana) fermions".

36 It is worth noticing that both Touschek (1948) and Serpe (1952) emphasized that starting from Fermi's theory of $\beta$ decay one can both treat the neutrino as Dirac or Majorana particle, so that one cannot conclude on the ground of the results of $\beta \beta$ emissions that neutrino is a Majorana particle. The only conclusion that can be reached is that Majorana theory can account for $\beta \beta$ decay. It was only with the development of the theory of weak interaction that new experiments could be designed in order to prove the nature of neutrino.

37 The merit of Racah (1937) was to show that Majorana's theory did not apply to the neutron because the latter could not have magnetic moment and its decays according to Majorana theory were against experimental evidence.

38 Furry underlined the relevance of Majorana's theory and explored the question of Lorentz invariance, as well as the possibility of subjecting neutrino to forces, finally identified with the nonelectric force of a scalar potential. In applying Majorana's formalism to the theory of $\beta$-radioactivity, Furry found that the physical interpretation is quite different from that of the ordinary theory, since only neutrinos appear instead of the neutrinos and antineutrinos of the usual picture. However he noticed that the results predicted for all observed processes should have been identical with those of the ordinary theory and therefore concluded that "an experimental decision between the formulation using neutrinos and antineutrinos and that using only neutrinos will apparently be even more difficult than the direct demonstration of the existence of the neutrino" (Furry 1938).
}

\section{The fate of Weyl's and Majorana's theories}

What happened to Majorana theory after 1937? Contrary to the case of Majorana (1932), there were immediate reactions to his paper on the symmetric theory of electron and positron. Both Racah $^{37}$ and Furry ${ }^{38}$ published two articles in 1937 and 1938, respectively, showing strengths and weaknesses associated to Majorana theory. Soon after the publication of Wigner (1939) and the beginning of WWII, however, there were other priorities and restricted publication policies in nuclear physics. Nevertheless, in Belgium $^{39}$ and The Netherlands, theoretical physicists did not stop investigating Majorana's proposal and Weyl's two-component theory. Among them, Hans Kramers and his colleagues were working on mesons (Dresden 2011), Weyl's two-component theory and the problem of representing neutrinos in Fermi's theory of $\beta$ decay also thanks to the research on spinors developed by van der Waerden in the 1930s. Apart from such a notable group and Pauli's (1941) mention of his neutrino theory, Majorana's work was apparently forgotten. I say apparently, because of course Majorana's colleagues and friends then working in the United States and Canada did not. Among them, especially, Enrico Fermi, Emilio Segrè and Bruno Pontecorvo contributed to the diffusion of Majorana's ideas by talking with colleagues and research assistants. Thus, Majorana theory of spinors and its application to neutrino simply underwent a silent phase in the 1940 s, a phase broken by some attempts encouraged by Kramers and his colleagues. ${ }^{40}$ Among these attempts considering both Majorana (1932) and Majorana (1937), there is Józef Kazimierz Lubański's (1941), who reported that Kramers himself suggested to him this work. Lubański also published Sur la théorie des particules élémentaires de spin quelconque. Partie I et Partie II in 1942, which represents a thorough study of Majorana (1932).

It is only with the end of the WWII and the beginning of the Cold War that a larger number of physicists rediscovered Majorana's work. It took time, however, to revitalize his 1937 theory and to start suggesting the right kinds of experiments to verify it. After WWII, certainly Bruno Touschek (1948) contributed to this aspect, together with other few attempts at discussing Majorana (1937) and its derivation from Weyl's two-component theory, such as Herbert Jehle's. Jehle joined the IAS in 1947/1948 and published a remarkable work on the two-component wave equations (see Jehle 1949) that was also mentioned by Case (1957) as a relevant study of Majorana equations for the neutrino. It assumed a certain relevance also Jean Serpe's (1952) that showed that Weyl's unquantized twocomponent wave equations were related to Majorana (1937) for $m=0$. He also found that in a Fock two-component representation, a Majorana free particle with $m=0$ can be described by Weyl's equations or by their complex conjugates, according to its spin state.

Therefore, as a preliminary result, one can conclude that there were at least two main reasons why Majorana two-component theory assumed a relevant role only in the 1950s and rather played a marginal role in the 1940s. First, one has to consider the development of experiments and facilities to detect the neutrino in the 1950s. The search for a confirmation of the existence of the

\footnotetext{
39 In particular Jean Serpe, who was a former pupil of L. Rosenfeld, became professor of theoretical physics in Liège. From 1941 he played an important role in discussing and disseminating Majorana's theory of neutrino, see Marage (2001). See also Gehéniau \& Serpe (1980, 85-94).

${ }^{40}$ Frederik Jozef Belinfante who published with Pauli On the statistical behaviour of known and unknown elementary particles in 1939 was also in Kramers' group. Kramers, Belinfante and Lubański (1941, esp. 616) took into account Majorana (1937). More in general the interest Kramers had on Majorana (1932) and Majorana (1937) can be ascribed to 1) the algebraic methodology displayed therein, which was affine to Kramers' and 2) to the fact that also Majorana applied Weyl's group theoretic approach to quantum mechanics.
} 
neutrino that arrived in 1956 by Cowan also assumed a political connotation during the Cold War, insofar as the USA and USSR largely competed in nuclear physics. Every theory of the neutrino was taken into account to design experiments, including Majorana's, and certainly the fact that one of the major experts in neutrino physics was Bruno Pontecorvo, a former colleague of Majorana in Rome, also played a role in promoting the diffusion of Majorana 1937 (see Pontecorvo 1982).

The second reason lies in the debate involving the scientific community of particle physicists on CPT symmetry (see Wick, Wightman and Wigner 1952; Yang 1986, 12-13) (1) $^{41}$ and the socalled theta-tau puzzle. In 1935, Yukawa predicted the existence of mesons, and only one year later, in 1936, physicists first detected the muon, which was thought to be a meson until 1947 when the existence of charged pions was experimentally proved. In the same year, George Rochester detected the kaon: the realm of particles was enlarging and the scenario of nuclear physics was far more complex than that of the early 1930s. Bearing this in mind, we can associate the revival of the study of two- and four-component theories of spinors to the increasing level of complexity encountered in the fundamental interactions and the subatomic world in the decade 1937-1947. The early-1950s were thus characterized by the effort of finding a general rule to classify new particles. For instance, during the Bagnéres-de-Bigorre conference in 1953 it was officially adopted the name "L-mesons" for pions and muons and "K-mesons" for the new particles with mass intermediate between the pion and the proton (see Shapiro 1953). With the increasing gathering of data on strange particles also grew evidence that $\mathrm{K}$ mesons possessed the approximate equal masses and lifetimes. In particular, the two particles $K_{\pi 3} \equiv \tau^{ \pm} \rightarrow \pi^{ \pm}+\pi^{+}+\pi^{-}$and $K_{\pi 2} \equiv \theta^{ \pm} \rightarrow \pi^{ \pm}+\pi^{0}$ seemed to have almost identical masses and lifetimes, but their parity properties seemed to be different thereby leading to the theta-tau puzzle. The theta and the tau possessed same charge, same mass, and indistinguishable lifetimes, yet they were not considered to be identical given that the theta could decay into two pions, showing a symmetric decay under mirror reflection, whereas the tau could decay into three pions, thereby showing an antisymmetric decay. Since it was taken for granted that parity was conserved in such decays, it was only assumed that the theta and the tau could not be the same particle.

Precisely the analogy between the theta-tau puzzle and the implications about parity of the two-component theories of Weyl and Majorana, led to their discussion at a large scale from mid-1950s onward. In April 1956, during the Sixth Annual Rochester Conference, a session was devoted to the "Theoretical Interpretation of New Particles". In that occasion, Oppenheimer noticed: "There are the five objects $K_{\pi 3}, K_{\pi 2}, K_{\mu 2}, K_{\mu 3}, K_{e 3}$. They have equal, or nearly equal, masses, and identical, or apparently identical, lifetimes. One tries to discover whether in fact one is dealing with five, four, three, two, or one particle. Difficult problems arise no matter what assumption is made" (Pais 1986, 281). During the very same session, Yang suggested experimental test and hypotheses to explain the

\footnotetext{
41 It is important to recall that in between 1954 and 1955 the CPT theorem received its proof by G. Lüders, W. Pauli and J. S. Bell.

${ }^{42} \mathrm{Wu}$ moved to Berkeley in 1936 and started working under the supervision of Emilio Segrè, a former member of Fermi's group in Rome and colleague of Majorana. She became the first woman instructor in physics at Princeton University in 1943 accepting a position offered by Prof. Henry Dewolf Smyth. She taught physics to naval officers (Lubkin 1971, 52-56; Chiang 2014, 74). When Wu came to Princeton she interacted with Pauli, later the two had a correspondence on the experiment on parity conservation (see Pauli 1979, 790; 796). From March 1944, she actively collaborated to the Manhattan Project, thereby moving to New York and starting working at Columbia on developing radiation detectors. After WWII, she worked at Columbia and became one of the leading experts in experiments on $\beta$ decay.
}

theta-tau puzzle and advanced in public the idea that the assumption of parity nonconservation could have been the key to overcome the difficulties. Lee and Yang (1956) suggested experiments to test their hypothesis that parity was not conserved in the weak interaction, for instance by means of the $\beta \beta$ decay of oriented nuclei. Precisely this was the experimental test performed by Chien-Shiung Wu and her collaborators in late 1956 (see Wu, Ambler, Hayward, Hoppes \& Hudson 1957). ${ }^{42}$ Even if Wu worked at Princeton in 1943, when Weyl was also there, the two did not interact that much. However, Wu probably knew about Weyl's two-component theory from his colleagues Lee and Yang. Yang, in particular, collaborated with Fermi in Chicago and in 1949 moved to the IAS where he became acquainted with both Weyl's and Majorana's theories, as well as with renormalization theory, pursued by Case, Dyson and Luttinger (see Yang 2005, 8-9). Based on Lee and Yang's claim (1956) that there was no empirical test supporting parity conservation in weak interaction, Wu and her team pursued the experiment that led to the discovery. After aligning ${ }^{60} \mathrm{Co}$ nuclei, they counted the number of decay electrons along the nuclear spin and opposite to the spin, and thereby found that a larger number of electrons were emitted in opposite direction to the spin than along the spin.

As underlined in Section 3, this approach considering parity nonconservation as a mathematical possibility to be tested is very much in the same spirit that guided Weyl in spelling out his theory and is also present in Lee and Yang (1957). In Parity nonconservation and a two-component theory of the neutrino, Lee and Yang claimed:

"In this theory (n.d. two-component theory of neutrino) the mass of the neutrino must be zero, and its wave function need only have two components instead of the usual four. That such a relativistic theory is possible is well known. It was, however, always rejected because of its intrinsic violation of space inversion invariance, a reason which is now no longer valid. (In fact, as we shall see later, in such a theory the violation of space inversion invariance attains a maximum)". (Lee and Yang 1957, 1671).

Lee and Yang (1957) notice at this point that if the twocomponent theory of the neutrino is correct, then this would represent a natural way to obtain the understanding of parity nonconservation in processes involving the neutrino. However, they also emphasized that in the case of the theta-tau puzzle, no neutrino was involved in the decay process of $K_{\pi 2}$ and $K_{\pi 3}$. In their view, all this pointed to a more fundamental level responding to the question of the origin of all weak interactions and to the nonaccidental character of parity nonconservation. ${ }^{43}$

It is time now to go back to one of the initial questions of this contribution and throw a fresh light on the role that Weyl's and Majorana's works played in the mid-1950s. Soon before and after Wu's results were released a number of articles on parity nonconservation in weak interaction (Salam 1957; Landau 1957a) and on Weyl's two-component theory and Majorana's theory of neutrino appeared (Landau 1957b; Case 1957; McLennan 1957 and Serpe 1957), thereby fostering the development of the V-A theory and enriching the new promising field of neutrino physics.

The most important work to analyze here for the purpose of the present paper is Case (1957) that proposed a new formulation of both Majorana and Weyl theories. At the time Kenneth Myron Case was Professor of Physics at the University of Michigan and affiliated

\footnotetext{
43 According to Yang $(2005,25)$, there were at least three reasons why parity nonconservation was not accepted before. First, space-time symmetry laws were assumed to be inviolable. Second, parity selection rules in nuclear physics and $\beta$ decay worked extremely well. Third, more importantly, nobody dissociated parity conservation in weak interactions from parity conservation in the strong interactions.
} 
to the IAS in Princeton. He reformulated Majorana theory for a particle with mass in terms of a two-component field, thereby showing that "the theory goes over continuously to the Weyl-type two-component equation as the mass tends to zero" (Case 1957). Furthermore, Case took into account that the asymmetries obtained in $\beta$ - and $\mu$-decay experiments only implied a small neutrino mass, and certainly not that its mass is zero. More importantly, he achieved to show what had been a mere suggestion of Salam (1957), Landau (1957a) and Lee and Yang (1957), namely he showed that the asymmetries, including $P$ nonconservation, are not implied by the mere use of a two-component theory or by the use of a fourcomponent one. It is rather "due to the fact that the interactions necessary to describe the physical world are not reflectioninvariant" (Case 1957). He deepened the similarity noticed by Lee and Yang (1957) concerning $\mathrm{K}$ particles and decays involving neutrinos and concluded that "the nonconservation of parity is not an intrinsic kinematical property of the neutrino but a dynamical property of the coupling" (Case 1957, 314). Furthermore, he added, as Lee and Yang (1957) did, that the question as to why parity is not conserved by pion interaction would seem neither more nor less deep than the corresponding question for neutrino interactions. Case therefore established the analogy between the twocomponent theory of neutrino and the theta-tau puzzle as follows:

"The use of a two-component neutrino theory may be a particularly elegant means of describing the parity nonconserving weak beta-decay interactions. The point is only that there is no logical connection between the two: the leftright asymmetries neither imply nor are necessarily implied by the use of a two-component theory" (Case 1957, 315).

Case clearly claimed that is rather the theory of weak interaction that implies certain relations between the neutrino mass, $\beta \beta$-decay and parity nonconservation. With Case (1957), thus, the scientific community of theoretical and experimental particle physicists "rediscovered" Weyl's and Majorana's theories by framing them within the new landscape of the making of V-A theory and the achievement of the CPT theorem. However, as shown in this contribution, scientific communities in Europe and United States never really stopped to discuss these theories, and therefore we should abandon the naïve idea that Weyl (1929b) and Majorana (1937) were suddenly "rediscovered" in 1957 just in order to offer a theoretical framework of decay processes involving neutrinos.

\section{Conclusive remarks}

"Everything comes to him who knows how to wait". Less than two years after Weyl's death, these words could have had appropriately expressed his impact on the history of physics after the discovery of the neutrino and parity violation in $\beta$-decay. Indeed, until the seesaw mechanism was established in the $1980 \mathrm{~s},{ }^{44}$ Weyl's equations were the standard way to describe neutrinos as zero rest mass particles. In more recent times, the search for experimental confirmation of neutrinoless double-beta decay still testifies the interest for Majorana's theory that was heavily influenced by Weyl. Furthermore, the implications of Majorana's theory are worth being

\footnotetext{
44 The seesaw mechanism was first proposed in between 1979 and 1980 by GellMann, Ramond and Slansky (1979), Yanagida (1980) and Mohapatra and Senjanovic (1980).

${ }^{45}$ In recent years, for instance, experiments involving institutions such as Princeton, Delft University and ETH-Zurich identified Majorana fermions in iron wires or topological superconductors (Nadj-Perge, Drozdov, Li, Chen \& Jeon, 2014) and Weyl fermions in semimetals (Soluyanov et al. 2015), thereby fostering expectations for the development of quantum computation.
}

investigated, not only because they develop a range of possibilities entailed in Weyl's, but also because its consequences could have a huge impact on other research fields, such as those related to superconductors and quantum computation. ${ }^{45}$ Finally, the present contribution aimed at showing that Lee, Yang and Wu interpreted at best the problem posed by Weyl (1929b) and Majorana (1937), namely they expressed at best the awareness that there was no direct experimental proof that parity was always conserved and that theoretically there can be found a mathematical description of its nonconservation in $\beta$-decay. This awareness grew with the discoveries of new particles in between 1935 and 1947 and led to the need of thinking of different parity properties for particles and antiparticles subject to different interactions. Weyl's legacy in the 1950 s was thus also a conceptual one regarding the methodology of scientific research and the importance of mathematics and its methods for the development of physics. By considering the history of alternative theories of spinors and the great experimental results obtained in 1956-1957 on parity nonconservation in $\beta$-decay, it has been shown how Weyl's 1929 theory was meant to answer to questions related to the debates on unified field theory, rather than to address the question of parity nonconservation. However, it also emerged that Weyl considered the violation of parity conservation as a mathematical possibility in the 1930s and that his theory was restored in the 1950 s through a complex process of circulation of knowledge.

\section{Acknowledgement}

The research leading to this article has been made possible thanks to the fellowship "Research in Paris 2013" sponsored by the Ville de Paris, the EC-FP7 PEOPLE-COFUND grant n. BP2013-100101, the research group 2014 SGR 1414 HIS-STM and the Mineco project HAR2014-57776. I am indebted to Gabriel Catren, Erhard Scholz and Christophe Eckes for fruitful discussions and grateful to an anonymous referee for insightful comments on earlier versions of this paper.

\section{References}

Amaldi, E. (1966). La vita e l'opera di E. Majorana. Accademia dei Lincei, Roma. Barbour, J. B., \& Vizgin, V. P. (2011). Unified Field Theories: In the first third of the 20th century. Springer.

Bonolis, L. (2004). From the Rise of the Group Concept to the Stormy Onset of Group Theory in the New Quantum Mechanics. A saga of the invariant characterization of physical objects, events and theories. Nuovo Cimento Rivista Serie, 27(4), $1-110$.

Brauer, R., \& Weyl, H. (1935). Spinors in n dimensions. American Journal of Mathematics, 425-449.

Budinich, P., \& Trautman, A. (2012). The spinorial chessboard. Springer.

Case, K. M. (1957). Reformulation of the Majorana theory of the neutrino. Physical Review, 107(1), 307.

Chiang, T.-C. (2014). "The First Lady of Physics Research." Madame Wu Chien-Shiung: The First Lady of Physics Research. World Scientific.

Coleman, R. A., \& Korté, H. (2001). Hermann Weyl: Mathematician, Physicist, Philosopher (Birkhäuser). In E. Scholz (Ed.), WorkHermann Weyl's Raum - Zeit Materie and a General Introduction to His Scientific (pp. 161-386). Basel.

Condon, E.U., \& Shortley, G.H., (1935). The theory of atomic spectra Cambridge University Press.

Cowan, C. L., Reines, F., Harrison, F. B., Kruse, H. W., \& McGuire, A. D. (1956). Detection of the free neutrino: a confirmation. Science, 124(3212), 103-104.

Darrigol, O. (1988). Statistics and combinatorics in early quantum theory. Historical Studies in the Physical and Biological Sciences, 17-80.

Darwin, C. G. (1927). The electron as a vector wave. Proceedings of the Royal Society of London. Series A, Containing Papers of a Mathematical and Physical Character, 116(773), 227-253.

Dirac, P. A. (1928). "The quantum theory of the electron". Part I. Proceedings of the Royal Society of London, 117(778), 610-624.

Dirac, P. A. (1928). "The quantum theory of the electron". Part II. Proceedings of the Royal Society of London, 118(779), 351-361.

Drago, A., \& Esposito, S. (2004). Following Weyl on quantum mechanics: the contribution of Ettore Majorana. Foundations of Physics, 34(5), 871-887.

Dresden, M. (2011). H. A. Kramers between tradition and revolution. Springer. 
Einstein, A. (1928). Riemann-Geometrie mit Aufrechterhaltung des Begriffes des Fernparallelismus (pp. 316-321). Wiley.

Einstein, A., \& Mayer, W. (1931). Systematische Untersuchung über kompatible Feldgleichungen, welche in einem Riemannschen Raume mit Fernparallelismus gesetzt werden können (pp. 365-374). Wiley.

Enz, C. P. (2002). No time to be brief: A scientific biography of Wolfgang Pauli. Oxford: Oxford University Press.

Fermi, E. (1934). Tentativo di una teoria dei raggi B. Il Nuovo Cimento (1924-1942), 11(1), 1-19.

Fock, V. (1929). Geometrisierung der Diracschen theorie des elektrons. Zeitschrift für Physik, 57(3-4), 261-277.

Fock, V., \& Iwanenko, D. (1929). Quantum geometry. Nature, 123, 838.

Fradkin, D. M. (1966). Comments on a paper by Majorana concerning elementary particles. American Journal of Physics, 34, 314.

French, S., \& Rickles, D. (2003). Understanding permutation symmetry. In Castellani, \& Brading (Eds.), Symmetries in Physics (pp. 212-238). Cambridge: Cambridge University Press.

Furry, W. H. (1938). Note on the theory of the neutral particle. Physical Review, $54(1), 56$.

Gehéniau \& Serpe. (1980). Esquisse de la contribution belge à la mécanique quantique, à ses prolongements et à des sujets connexes. Florilège des Sciences II Brussel, 1980, 85-94.

Gelfand, M., \& Yaglom, A. M. (1948). General Relativistic-Invariant Equations and Infinite-Dimensional Representations of the Lorentz Group (in Russian. In Zh. Eksperim. I Theor. Fiz., 18 pp. 707-733) (English transl.: TT-345, National Research Council of Canada (1953)](.).

Gell-Mann, M., Ramond, P., \& Slansky, R. (1979). Complex Spinors and Unified Theories. In P. van Nieuwenhuizen, \& D. Z. Freedman (Eds.), Supergravity. Amsterdam: North Holland.

Giulini, D. (2009). Concepts of symmetry in the work of Wolfgang Pauli. In H. Atmanspacher, \& H. Primas (Eds.), Recasting Reality (pp. 33-82). Springer Berlin Heidelberg.

Goldstein, C., \& Ritter, J. (2003). The varieties of unity: Sounding unified theories 1920-1930. In A. Ashtekar, et al. (Eds.), Revisiting the Foundations of Relativistic Physics (pp. 93-149). Dordrecht: Springer.

Gönner, H. F. (2004). On the history of unified field theories. Living reviews in relativity, $7(1), 2$.

Hoefer, C. (2000). Kant's hands and Earman's pions: Chirality arguments for substantival space. International Studies in the Philosophy of Science, 14(3), 237-256.

Infeld, L., \& van der Waerden, B.L. (1933). Die Wellengleichung des Elektrons in der allgemeinen Relativitätstheorie. Verlag d. Akad. d. Wissenschaften.

Jehle, H. (1934). Zur allgemeinen relativistischen Quantenmechanik. Zeitschrift für Physik, 87, 370-374.

Jehle, H. (1935). Zur allgemein-relativistischen Quantenmechanik. Zeitschrift für Physik, 94, 692-706.

Jehle, H. (1949). Two-component wave equations. Physical Review, 75(10), 1609.

Kragh, H. (1981). The genesis of Dirac's relativistic theory of electrons. Archive for history of exact sciences, 24(1), 31-67.

Kragh, H. (1990). Dirac: A Scientific Biography. Cambridge University Press.

Kramers, H. A., Belinfante, F. J., \& Lubański, J. K. (1941). Über freie Teilchen mit nichtverschwindender Masse und beliebiger Spinquantenzahl. Physica, 8, 597-627.

Landau, L. (1957). On the conservation laws for weak interactions. Nuclear Physics, 3(1), 127-131.

Landau, L. (1957). Об одной возмоЖности для поляризационных свойств нейтрино [On one possibility for polarization properties of the neutrino]. Zh. Eksp. Teor. Fiz, 32, 407[JETP 5 (1957) 337] Reprinted. In P. K. Kabir (Ed.), The Development of Weak Interaction Theory. New York - London: Gordon and Breach Science Publishers $(1963)(, 67)$.

Laporte, O. (1924). Die Struktur des Eisenspektrums. Zeit Physics, 23, 135.

Lee, T. D., \& Yang, C. N. (1956). Question of parity conservation in weak interactions. Physical Review, 104(1), 254.

Lee, T. D., \& Yang, C. N. (1957). Parity nonconservation and a two-component theory of the neutrino. Physical Review, 105(5), 1671.

Lubkin, G. B. (1971). Women in physics. Physics Today, 24, 23.

Lubański, J. K. (1941). Sur le spin des particules élémentaires. Physica, 8(1), 44-52.

Majorana, E. (1937). Symmetric theory of electron and positron (2006). In G. F. Bassani (Ed.), Ettore Majorana Scientific Papers (pp. 218-231). Bologna and Berlin: SIF and Springer.

Majorana, E. (1932). Relativistic theory of particles with arbitrary intrinsic angular momentum (2006). In G. F. Bassani (Ed.), Ettore Majorana Scientific Papers (pp. 273-275). Bologna and Berlin: SIF and Springer.

Marage, P. (2001). La physique nucléaire et la physique des particules élémentaires. Histoire des sciences en Belgique, 1815-2000, De Robert Halleux (ed.), Bruxelles, Dexia/La Renaissance du Livre.

McLennan, J. A., Jr (1957). Parity nonconservation and the theory of the neutrino. Physical Review, 106(4), 821.

Mehra, J., \& Rechenberg, H. (2001). The conceptual completion and the extensions of quantum mechanics 1932-1941; Epilogue: aspects of the further development of quantum theory 1942-1999. Springer.

Mohapatra, R. N., \& Senjanovic, G. (1980). Neutrino mass and spontaneous parity violation. Physical Review Letters, 44, 912.

Nadj-Perge, S., Drozdov, I. K., Li, J., Chen, H., Jeon, S., et al. (2014). Observation of Majorana fermions in ferromagnetic atomic chains on a superconductor. Science, 346(6209), 602-607.
Ne'eman, Y. (1978). Spinor-type fields with linear, affine and general coordinate transformations. In Annales de l'IHP Physique théorique, 28(4), 369-378.

O'Raifeartaigh, L., \& Straumann, N. (2000). Gauge theory: Historical origins and some modern developments. Reviews of Modern Physics, 72(1), 1.

Pashby, T. (2012). Dirac's prediction of the positron: a case study for the current realism debate. Perspectives on Science, 20(4), 440-475.

Pais, A. (1986). Inward Bound: On Matter and Forces in the Physical World. Oxford University Press.

Pauli, W. (1927). Zur Quantenmechanik des magnetischen Elektrons. In: Zeitschrift für Physik 43 601-623.

Pauli, W. (1933). Die allgemeinen Prinzipien der Wellenmechanik. Handbuch der Physik (pp. 83-272). Berlin: Springer.

Pauli, W. (1941). Relativistic field theories of elementary particles. Reviews of Modern Physics, 13(3), 203.

Pauli, W. (1961). "Zur älteren und neueren Geschichte des Neutrinos". In: Aufsätze und Vorträge über Physik und Erkenntnistheorie (pp. 156-180). Vieweg: Teubner Verlag.

Pauli, W. (1979) (1919-1929). In A. Hermann (Ed.), Scientific Correspondence with Bohr, Einstein, Heisenberg (Volume 1). Dordrecht: Springer.

Pickering, A. (1990). Reason enough? More on parity-violation experiments and electroweak gauge theory. In PSA: Proceedings of the biennial meeting of the philosophy of science association pp. 459-469.

Pontecorvo, B. (1957). Mesonium and antimesounium. Journal of Experimental and Theoretical Physics, 33, 549 (1957)( [Sov. Phys. JETP 6, 429 (1958)].

Pontecorvo, B. (1963). The neutrino and its role in astrophysics. Physics-Uspekhi $6(1), 1-12$.

Pontecorvo, B. (1982). The infancy and youth of neutrino physics: Some recollections. Le Journal de Physique Colloques, 43(C8) (C8-221-236).

Pontecorvo, B. (1983). Pages in the development of neutrino physics. Soviet Physics Uspekhi, 26(12), 1087.

Pooley, O. (2003). Handedness, parity violation, and the reality of space. In K. Brading, \& E. Castellani (Eds.), Symmetries in Physics: Philosophical Reflections (pp. 250-280). Cambridge: Cambridge University Press.

Racah, G. (1937). Symmetry between particles and anti-particles. Nuovo cimento, 14 $322-328$.

Recami E. (1991). Il caso Majorana.Epistolario, documenti, testimonianze. Mondadori: Milan.

Ritter, J. (2011). Geometry as physics: Oswald Veblen and the Princeton school. In K. H. Schlote, \& M. Schneider (Eds.), Mathematics meets Physics. A Contribution to their Interaction in the 19th and the First Half of the 20th Century (pp. 145-179). Berlin: Springer.

Roqué, X. (1997). The manufacture of the positron. Studies in History and Philosophy of Modern Physics, 28(1), 73-129.

Salam, A. (1957). On parity conservation and neutrino mass. Il Nuovo Cimento, 5(1), 299-301.

Shapiro, M. M. (1953). International congress on cosmic radiation at Bagneres de Bigorre. Science, 118(3076), 701-707.

Scholz, E. (2005). Local spinor structures in V. Fock's and H. Weyl's work on the Dirac equation (1929). In J. Kouneiher (Ed.), Géométrie au XXe siècle, 1930-2000: histoire et horizons (pp. 284-301). Montréal: Presses internationals Polytechnique.

Scholz, E. (2006). Introducing groups into quantum theory (1926-1930). Historia Mathematica, 33(4), 440-490.

Schouten, J. A. (1931). Dirac equations in general relativity. Journal of Mathematical Physics, 10, 239.

Schweber, S. S. (1994). QED and the men who made it: Dyson, Feynman, Schwinger and Tomonaga. Princeton: Princeton University Press.

Segrè, E. (1970). Enrico Fermi, Physicist. University of Chicago Press.

Serpe, J. (1952). Sur la théorie abrégée des particules de spin 1/2. Physica, 18(5), 295-306.

Serpe, J. (1957). Remarks on parity conservation and the two-component theory of the neutrino. Nuclear Physics, 4, 183-187.

Soluyanov, A. A., Gresch, D., Wang, Z., Wu, Q., Troyer, M., Dai, X., \& Bernevig, B. A (2015). Type-II Weyl semimetals. Nature, 527(7579), 495-498.

Speiser, A. (1923). Theorie der Gruppen von endlicher Ordnung, Berlin.

Straumann, N. (1997). Weyl's classic 1929. In L. O'Raifeartaigh (Ed.), The Dawning of Gauge Theory (pp. 107-120). Princeton: Princeton University Press.

Straumann, N. (2001). Ursprünge der Eichtheorien. In E. Scholz (Ed.), Hermann Weyl's Raum-Zeit-Materie and a General Introduction to His Scientific Work (pp. 138-155). Birkhäuser: Basel.

Touschek, B. (1948). Zur Theorie des doppelten $\beta$-Zerfalls. Zeitschrift für Physik $A$ Hadrons and Nuclei, 125(1), 108-132.

Uhlenbeck, G. E., \& Goudsmit, S. (1925). Ersetzung der Hypothese vom unmechanischen Zwang durch eine Forderung bezüglich des inneren Verhaltens jedes einzelnen Elektrons. Naturwissenschaften, 13(47), 953-954.

van der Waerden, B. L. (1929). Spinoranalyse. Nachrichten von der Gesellschaft der Wissenschaften zu Göttingen. Mathematisch-Physikalische Klasse, 100.

Vaz, J., Jr, \& Da Rocha, R., Jr (2016). An Introduction to Clifford Algebras and Spinors. Oxford: Oxford University Press.

Veblen, O. (1933). Geometry of four-component Spinors. Proceedings of the National Academy of Sciences USA, 19, 503-517.

Veblen, O. (1933). Geometry of two-component Spinors. Proceedings of the National Academy of Sciences USA, 19, 462-474.

Veblen, O. (1933). Spinors in Projective Relativity. Proceedings of the National Academy of Sciences USA, 19, 979-989. 
Veblen, O. (1937). Spinors and projective geometry. In International Congress of Mathematicians, Oslo, Vol. 1, pp. 111-127.

Weyl, H. (1929a). Gravitation and the electron. Proceedings of the National Academy of Sciences, 15(4), 323-334

Weyl, H. (1929). Elektron und Gravitation. Zeitschrift für Physik, 56(5), 330-352.

Weyl, H. (1929). "Consistency in mathematics". The Rice Institute Pamphlets (16), pp. 245-265 (1968). In K. Chandrasekharan (Ed.), Hermann Weyl: Gesammelte Abhandlungen, 4 pp. 150-169). Berlin, Heidelberg, New York: Springer.

Weyl, H. (1931). Gruppentheorie und Ouantenmechanik (Hirzel, Leipzig 1928); English translation by H.P. Robertson. Theory of Groups and Quantum Mechanics. London: Methuen.

Wick, G. C., Wightman, A. S., \& Wigner, E. P. (1952). The intrinsic parity of elementary particles. Phys Rev, 88, 101.

Wiener, N., \& Struik, D. J. (1927). Quantum theory and gravitational relativity. Nature, $119,853-854$.

Wigner, E. (1927). Einige Folgerungen aus der Schrödingerschen Theorie für die Termstrukturen. Zeit Physics, 43, 624.
Wigner, E. (1939). On unitary representations of the inhomogeneous Lorentz group. Annals of Mathematics, 149-204.

Wroblewski, A. K. (2008). The downfall of parity-the revolution that happened fifty years ago. Acta Physica Polonica B, 39(2).

Wu, C. S., Ambler, E., Hayward, R. W., Hoppes, D. D., \& Hudson, R. P. (1957) Experimental test of parity conservation in beta decay. Physical Review, 105(4), 1413-1415.

Yanagida, T. (1980). Horizontal symmetry and masses of neutrinos. Progress of Theoretical Physics, 64(3), 1103-1105.

Yang, C.N. (1986). Hermann Weyl's Contribution to Physics. In Chandrasekharan, K., Yang, C. N., Penrose, R., \& Borel, A. (1986). Hermann Weyl: 1885-1985: Centenary Lectures. Springer, pp. 7-21.

Yang, C. N. (2005). Selected Papers (1945-1980), with Commentary, 36. World Scientific.

Zaycoff, R. (1929). Derivation of the Dirac-Whittaker wave equations from Einstein's unified field theory. Zeit Phys, 56, 862-864. 